\title{
Energy and Time Distribution of Photoelectrons Released in Water under Ultra-Short X-Ray Pulse Irradiation
}

\author{
W. BŁACHUCKI \\ Institute of Nuclear Physics, Polish Academy of Sciences, 31-342 Kraków, Poland
}

\begin{abstract}
In the probe-before-destroy methodology, developed with the advent of X-ray free-electron lasers, the investigated system is probed with an ultra-short (femtosecond) X-ray pulse within time shorter than that the X-rayinduced damage needs to propagate. Radiation damage is typically considered in terms of the Coulomb explosion which needs about 50 fs to induce observable effects in the material structure. It has been, however, shown that the much faster electronic structure change has also a significant influence on the X-ray emission spectroscopy data and may alter the X-ray diffraction patterns. This work reports on energy and time distribution of photoelectrons induced in water by an X-ray pulse with properties typical for an X-ray free-electron laser operated in the intensity regime below the sequential photoionization regime. The electron flux was simulated on the basis of a kinetic model and was found to be significant over the course of the X-ray pulse duration. The presented findings call for consideration of the X-ray-induced electrons in studies on solutions and samples embedded in matrices.
\end{abstract}

DOI: 10.12693/APhysPolA.137.13

PACS/topics: X-ray free electron laser, X-ray induced damage

\section{Introduction}

Since the work of Blake and Phillips [1] uncovering the damaging effect of X-ray irradiation on crystalline proteins, X-ray studies on complex systems has been accompanied by a concern about the damage done by the incident beam to the studied material, especially when using the bright 3rd and 4th generation radiation sources (see, e.g., Refs. [2-6]). The radiation damage is the unwanted consequence of the high ionization of the studied material by the intense probe beam: electronic structure change, bond breaking, the Coulomb explosion, structural change. This phenomenon naturally refers primarily to the X-ray radiation damage and is crucial in the case of study on chemical complexes and biological systems as it does not allow an intact target material characterization. Until now different approaches have been developed to profit from the high peak brightness of the pulsed X-ray sources without losing the sought information on the studied system. The X-ray damage can be overcome to a certain extent by placing the studied material in a flowing liquid jet $[7,8]$. It ensures that each incident X-ray pulse interacts with a fresh sample of the target, unaffected by the potential radicals released through the damage done by the previous pulse. Another tool of preventing the radiation damage influence on the measured data was brought with the development of cryocooling techniques [9-11]. Cooling the target material down to very low temperatures (of about $100 \mathrm{~K}$ ) slows down the free radicals diffusion which allows extending

corresponding author; e-mail: wojciech.blachucki@ifj.edu.pl the time for data collection or equivalently increasing the limit of acceptable radiation dose. Despite the radiation damage prevention techniques used, in certain applications additional attenuation of too intense incident photon beam is necessary.

A progress in overcoming the X-ray damage influence on the experimental data has been made possible on the part of the excitation beam itself with the advent of the 4th generation X-ray sources, X-ray free-electron lasers (XFELs). The extremely bright XFEL sources, $\sim 10^{10}$ brighter than synchrotrons, produce femtosecondshort X-ray pulses reaching peak intensity on a target of the order of $10^{20} \mathrm{~W} / \mathrm{cm}^{2}$ and photon fluxes of about $10^{34}$ photon $/\left(\mathrm{cm}^{2} \mathrm{~s}\right)$ and beyond $[12,13]$. These unique properties allow studying new fundamental processes in atomic and radiation physics (see, e.g., Refs. [14-17]) and in the case of more complex systems make timeresolved measurements on femtosecond molecular processes possible $[18,19]$. It is shortening the X-ray pulse duration to femtoseconds, while maintaining high number of photons that opened the way to develop the socalled probe-before-destroy approach [20-29] allowing to probe molecular systems before the radiation damage causes observable changes in them. It was demonstrated, mainly by means of the X-ray diffraction (XRD) method, that this technique works with a wide range of pulse durations, with the minimal one of about $50 \mathrm{fs}$, where the photon flux was of the order of $10^{32}$ photon $/\left(\mathrm{cm}^{2} \mathrm{~s}\right)$ [28].

The radiation damage is typically considered in terms of the Coulomb explosion leading to a structural change while it has been shown that the much faster electronic structure change has also a significant influence on the X-ray emission spectroscopy (XES) data [30] and can potentially affect the X-ray diffraction (XRD) results $[31,32]$. This work is focused on a study of 
energy and time distribution of photoelectrons released in a water target irradiated with an ultra-short X-ray pulse. The distribution was simulated using a kinetic model for the photon flux of $2.2 \times 10^{32}$ photon $/\left(\mathrm{cm}^{2} \mathrm{~s}\right)$, thus much below the sequential photoionization domain [33]. The results show that penetration of the sample by X-ray photons is accompanied by a significant flux of energetic electrons which can interact with the medium over the course of the pulse duration. The described effect must be taken into consideration in most of the studies done at XFELs with samples diluted in the water environment or enclosed in soft matrices, as the photoelectrons released can interact with the target sample and modify the electronic configuration of the target atom, possibly before the interaction with an X-ray photon from the incident beam.

\section{Method}

The photoelectrons of energy $E$ ejected from water molecules in the photoionization events undergo inelastic collisions with the $\mathrm{H}_{2} \mathrm{O}$ molecules at the average frequency per electron $f_{e}(E)[34,35]$ :

$$
f_{e}(E)=n_{a} \sigma_{i, e \leftrightarrow a}(E) \bar{v}_{e}(E),
$$

where $n_{a}$ is the number of atoms $a$ per unit volume, $\sigma_{i, e \leftrightarrow a}(E)$ is the cross-section for inelastic interaction of an electron with an atom $a$, and $\bar{v}_{e}(E)$ is the average electron speed. Inelastic collisions result in electron energy losses, which makes both $E$ and $E$-dependent quantities evolve in time $t$. With the average energy loss per interaction $\bar{E}_{\text {loss }, e \leftrightarrow a}(E)$ the temporal evolution of an electron's energy $E$ can be described with the following differential equation:

$$
\mathrm{d} E=-f_{e}(E) \bar{E}_{\text {loss }, e \leftrightarrow a(E)} \mathrm{d} t .
$$

Introducing the stopping power defined by Inokuti [36]:

$$
S_{a}(E)=\frac{\mathrm{d} E_{\text {loss }, e \leftrightarrow a}}{\mathrm{~d} z}=n_{a} \sigma_{i, e \leftrightarrow a} \bar{E}_{\text {loss }, e \leftrightarrow a(E)},
$$

where $S_{a}(E)$ denotes the linear stopping power for atoms $a$ and $\mathrm{d} E_{l o s s, e \leftrightarrow a} / \mathrm{d} z$ represents a derivative of electron energy loss over distance travelled in a medium, Eq. (2.1) and Eq. (2.2) can be combined in the formula:

$$
\mathrm{d} E=-S_{a}(E) \bar{v}_{e}(E) \mathrm{d} t .
$$

Energy and time distributions of the energetic electrons $n_{e}(E, t)$ were calculated using Eq. (2.4) and the incident X-ray pulse's time distribution was described with a Gaussian of the full width at half maximum (FWHM) of $30 \mathrm{fs}$ and with the total number of photons per pulse of $4 \times 10^{11}$. The target was described as a planar water jet of the mass density of $1 \mathrm{~g} / \mathrm{cm}^{3}$ and of the thickness of $0.1 \mathrm{~mm}$. The calculation was done for the irradiation area of $6 \mu \mathrm{m}^{2}$ and for three energies of incident photons: 4,6 , and $8 \mathrm{keV}$. The released electrons were assumed not to escape the irradiated volume. In the simulation the partial photoionization cross-sections from Ref. [37] were used and stopping powers for $\mathrm{H}_{2} \mathrm{O}$ were taken from Ref. [38].

\section{Results and discussion}

In the simulation only the electrons of energy above $12 \mathrm{eV}$ were considered due to the lack of precise stopping power of data below this limit. The energy and time distributions of photoelectrons simulated for three energies of incident photons are presented in Fig. 1a-c. For the three studied photon energies 4,6 , and $8 \mathrm{keV}$, respectively 82,24 , and $10 \%$ of photons were absorbed by the $\mathrm{O}$ atoms inducing a considerable flux of photoelectrons which at certain moments, especially for softer X-ray irradiation, exceeded the flux of incident photons. The electrons penetrate the medium losing their energy gradually over the course of the pulse duration which results in a very broad electron spectrum at every moment. Figure 1d presents electron spectra at the moment of photon pulse maximum ( $0 \mathrm{fs}$ ) for the three incident photon energies studied. As shown, the lower is the photon energy, the more electrons are released in the sample. The electron pileup appearing at the lowest energies arises from the small electron stopping power for water in this energy range.

The effect of stronger photoionization for less energetic X-rays is better illustrated in Fig. 2a showing how the total electron flux evolves in time for different incident photon energies. The presented electron curves tend to have Gaussian-like profiles with maxima at tens of fs after the X-ray pulse maximum. It is worth noting that, as it is shown in Fig. 2a, the total number of electrons
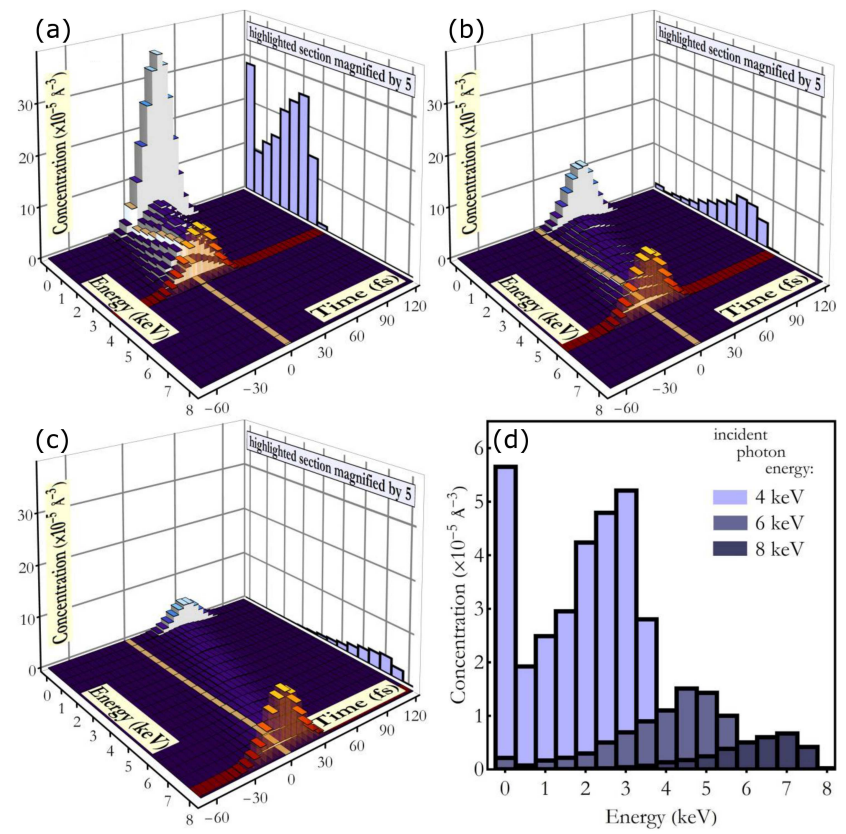

Fig. 1. (a, b, c) Energy and time distributions of induced photoelectrons (blue bars) simulated for three energies of the incident X-rays: 4,6 , and $8 \mathrm{keV}$, respectively. The Gaussian profiles of X-ray pulses are also shown (red bars). The data were integrated over 5 fs time periods and $500 \mathrm{eV}$ energy intervals. (d) The sections highlighted in (a), (b), and (c). 

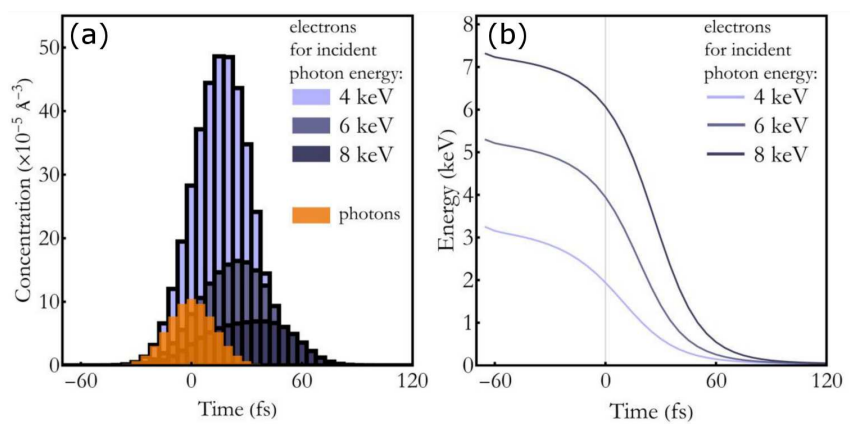

Fig. 2. (a) Temporal evolution of the total number of electrons and photons per unit volume. (b) Mean electron energy as a function of time. The gray line indicates the moment of the X-ray pulse maximum intensity.

penetrating the sample at a given moment may be greater than number of photons irradiating the material at the same moment. It is due to the fact that electrons interact with the medium multiple times losing the energy gradually (see Fig. 2b), while interaction of each photon has a binary character.

Despite the total number of photoelectrons being smaller than that of the incident photons, the electrons can take a significant part in ionization of the sample dissolved in water. It was shown for example in a work done at an XFEL on hexacyanoferrate(II) groups in a water solution [30]. The authors showed that Fe atoms were ionized by photo- and Auger electrons at average frequency which was half of the photoionization rate and estimated that about $5 \%$ of Fe atoms were already ionized by electrons prior to absorbing the probe photon. This is possible due to the fact that, unlike photons, electrons circulate in the material longer and the crosssection for electron-impact is much larger than the photoionization cross-section. A few $\mathrm{keV}$ electrons, with the average travel range between collisions in water of about 20-30 nm [39] and average travel time of less than $1 \mathrm{fs}$, may ionize the target atoms within the irradiated volume and the pulse duration limits at a frequency comparable to that of photons.

\section{Conclusions}

In this work typical XFEL experimental conditions were adopted to simulate energy and time distribution of photoelectrons released in a water target irradiated with an ultra-short X-ray pulse. The amount of energetic electrons was estimated to be comparable or exceeding that of the incident photons, particularly for soft X-rays. Analysis of X-ray-induced electron flux including Auger electrons and its impact on the material ionization were described elsewhere (see, e.g., Ref. [30]). The presented results indicate a potential necessity of considering in $\mathrm{X}$-ray studies the electron flux induced in the investigated material by the probe X-ray beam, even with a XFEL beam of ultra-short pulses. The energetic electrons may significantly alter the electronic structure of the material within time shorter than 50 fs needed for atoms movement [28], and affect XES [30] and XRD [31, 32] data. The described effects may be of particular importance in experiments on solutions and on embedded samples.

\section{Acknowledgments}

This work was supported by the National Science Centre (Poland) under grant no. 2017/27/B/ST2/01890. I wish to acknowledge Dr. hab. Jakub Szlachetko from the Institute of Nuclear Physics of the Polish Academy of Sciences in Kraków (Poland) for his valuable comments made during the preparation of this work.

\section{References}

[1] C.C.F. Blake, D.C. Phillips, in: Proc. Symp. on the Biological Effects of Ionizing Radiation at the Molecular Level, International Atomic Energy Agency, Vienna 1962, p. 183.

[2] R. Henderson, Quarterly Rev. Biophys. 28, 171 (1995).

[3] M. Weik, R.B.G. Ravelli, G. Kryger, S. McSweeney, M.L. Raves, M. Harel, P. Gros, I. Silman, J. Kroon, J.L. Sussman, Proc. Natl. Acad. Sci. 97, 623 (2000).

[4] S.M. Polvino, C.E. Murray, O. Kalenci, I.C. Noyan, B. Lai, Z. Cai, Appl. Phys. Lett. 92, 224105 (2008).

[5] E.K. Curwood, H.M. Quiney, K.A. Nugent, Phys. Rev. A 87, 053407 (2013).

[6] A. Gianoncelli, L. Vaccari, G. Kourousias, D. Cassese, D.E. Bedolla, S. Kenig, P. Storici, M. Lazzarino, M. Kiskinova, Sci. Rep. 5, 10250 (2015).

[7] M. Faubel, S. Schlemmer, J.P. Toennies, Zeitschr. Phys. D At. Mol. Clust. 10, 269 (1988).

[8] M. Faubel, B. Steiner, J.P. Toennies, J. Chem. Phys. 106, 9013 (1998).

[9] D.J. Haas, M.G. Rossmann, Acta Crystallogr. B 26, 998 (1970).

[10] D.W. Rodgers, Meth. Enzymol. 276, 183 (1997).

[11] E.F. Garman, R.L. Owen, Acta Crystallogr. D 62, 32 (2006).

[12] H. Yumoto, H. Mimura, T. Koyama, S. Matsuyama, K. Tono, T. Togashi, Y. Inubushi, T. Sato, T. Tanaka, et al., Nat. Photon. 7, 43 (2013).

[13] H. Mimura, H. Yumoto, S. Matsuyama, T. Koyama, K. Tono, Y. Inubushi, T. Togashi, T. Sato, J. Kim, R. Fukui, et al., Nat. Commun. 5, 3539 (2014).

[14] L. Young, E.P. Kanter, B. Kr'́assig, Y. Li, A.M. March, S.T. Pratt, R. Santra, S.H. Southworth, N. Rohringer, L.F. DiMauro, G. Doumy, et al., Nature 466, 56 (2010).

[15] K. Tamasaku, E. Shigemasa, Y. Inubushi, T. Katayama, K. Sawada, H. Yumoto, H. Ohashi, H. Mimura, M. Yabashi, K. Yamauchi, T. Ishikawa, Nat. Photon. 8, 313 (2014).

[16] N. Rohringer, D. Ryan, R.A. London, M. Purvis, F. Albert, J. Dunn, J.D. Bozek, C. Bostedt, A. Graf, R. Hill, S.P. Hau-Riege, J.J. Rocca, Nature 481, 488 (2012). 
[17] S.M. Vinko, O. Ciricosta, B.I. Cho, K. Engelhorn, H.-K. Chung, C.R.D. Brown, T. Burian, J. Chalupský, R.W. Falcone, C. Graves, et al., Nature 482, 59 (2012).

[18] H.T. Lemke, C. Bressler, L.X. Chen, D.M. Fritz, K.J. Gaffney, A. Galler, W. Gawelda, K. Haldrup, R.W. Hartsock, H. Ihee, et al., J. Phys. Chem. A 117, 735 (2013).

[19] M. Beye, T. Anniyev, R. Coffee, M. Dell'Angela, A. Föhlisch, J. Gladh, T. Katayama, S. Kaya O. Krupin, A. Møgelhøj, et al., Phys. Rev. Lett. 110, 186101 (2013).

[20] H.N. Chapman, P. Fromme, A. Barty, T.A. White, R.A. Kirian, A. Aquila, M.S. Hunter, J. Schulz, D.P. DePonte, U. Weierstall, et al., Nature 470, 73 (2011).

[21] M.S. Hunter, D.P. DePonte, D.A. Shapiro, R.A. Kirian, X. Wang, D. Starodub, S. Marchesini, U. Weierstall, R.B. Doak, J. C.H. Spence, P. Fromme, Biophys. J. 100, 198 (2011).

[22] R.A. Kirian, T.A. White, J.M. Holton, H.N. Chapman, P. Fromme, A. Barty, L. Lomb, A. Aquila, F.R.N.C. Maia, A.V. Martin, R. Fromme, X. Wang, M.S. Hunter, K.E. Schmidt, J.C.H. Spence, Acta Crystallogr. A 67, 131 (2011)

[23] A. Barty, C. Caleman, A. Aquila, N. Timneanu, L. Lomb, T.A. White, J. Andreasson, D. Arnlund, S. Bajt, T.R.M. Barends, et al., Nat. Photon. 6, 35 (2012)

[24] L. Lomb, T.R.M. Barends, S. Kassemeyer, A. Aquila, S.W.Epp, B. Erk, L. Foucar, R. Hartmann, B. Rudek, D. Rolles, et al., Phys. Rev. B 84, 214111 (2011).

[25] A. Aquila, M.S. Hunter, R.B. Doak, R.A. Kirian, P. Fromme, T.A. White, J. Andreasson, D. Arnlund, S. Bajt, T.R.M. Barends, et al., Opt. Expr. 20, 2706 (2012).

[26] L.C. Johansson, D. Arnlund, T.A. White, G. Katona, D.P. DePonte, U. Weierstall, R.B. Doak, R.L. Shoeman, L. Lomb, E. Malmerberg, et al., Nat. Meth. 9, 263 (2012).
[27] R. Koopmann, K. Cupelli, L. Redecke, K. Nass, D.P. DePonte, T.A. White, F. Stellato, D. Rehders, M. Liang, J. Andreasson, et al., Nat. Meth. 9, 259 (2012).

[28] J. Kern, R. Alonso-Mori, J. Hellmich, R. Tran, J. Hattne, H. Laksmono, C. Glöckner, N. Echols, R.G. Sierra, J. Sellberg, et al., Proc. Natl. Acad. Sci. 109, 9721 (2012).

[29] R. Alonso-Mori, J. Kern, R.J. Gildea, D. Sokaras, T.-C. Weng, B. Lassalle-Kaiser, R. Tran, J. Hattne, H. Laksmono, J. Hellmich, et al., Proc. Natl. Acad. Sci. 109, 19103 (2012).

[30] W. Błachucki, Y. Kayser, J. Czapla-Masztafiak, M. Guo, P. Juranić, M. Kavčič, E. Källman, G. Knopp, M. Lundberg, C. Milne, J. Rehanek, J. Sá, J. Szlachetko, Struct. Dyn. 6, 024901 (2019).

[31] S.P. Hau-Riege, Phys. Rev. A 76, 042511 (2007).

[32] K. Nass, L. Foucar, T.R.M. Barends, E. Hartmann, S. Botha, R.L. Shoeman, R.B. Doak, R. Alonso-Mori, A. Aquila, S. Bajt, et al., J. Synchrotr. Rad. 22, 225 (2015).

[33] A. Rudenko, L. Inhester, K. Hanasaki, X. Li, S.J. Robatjazi, B. Erk, R. Boll, K. Toyota, Y. Hao, O. Vendrell, et al., Nature 546, 129 (2017).

[34] P.M. Banks, Planet. Space Sci. 14, 1085 (1966).

[35] A.V. Vinogradov, V.P. Shevel'ko, Sov. Phys. JETP 44, 542 (1976)

[36] M. Inokuti, Rev. Mod. Phys. 43, 297 (1971).

[37] T. Schoonjans, A. Brunetti, B. Golosio, M.S. del Rio, V.A. Solé, C. Ferrero, L. Vincze, Spectrochim. Acta $B$ 66, 776 (2011).

[38] Z. Francis, S. Incerti, M. Karamitros, H.N. Tran, C. Villagrasa, Nucl. Instrum. Methods Phys. Res. B 269, 2307 (2011)

[39] H. Shinotsuka, B. Da, S. Tanuma, H. Yoshikawa, C.J. Powell, D.R. Penn, Surf. Interface Anal. 49, 238 (2016). 